\title{
Factors associated with intensive care admission in patients with lung cancer: a population-based observational study of 26, 731 patients
}

Kathryn Puxty ${ }^{1 *}$, Christopher H. Grant ${ }^{2,1}$, Philip McLoone ${ }^{2}$, Billy Sloan², Tara Quasim¹, Kate Hulse ${ }^{1}$ and David S. Morrison ${ }^{2}$

\begin{abstract}
Background: Lung cancer is the most common cause of cancer related death worldwide and survival is poor. Patients with lung cancer may develop a critical illness, but it is unclear what features are associated with an Intensive Care Unit (ICU) admission.

Methods: This retrospective, observational, population-based study of linked cancer registration, ICU, hospital discharge and mortality data described the factors associated with ICU admission in patients with lung cancer. The cohort comprised all incident cases of adult lung cancer diagnosed between 1st January 2000 and 31st December 2009 in the West of Scotland, UK, who were subsequently admitted to an ICU within 2 years of cancer diagnosis. Multiple logistic regression was used to determine factors associated with admission.

Results: 26,731 incident cases of lung cancer were diagnosed with 398 (1.5\%) patients admitted to an ICU. Patients were most commonly admitted with respiratory conditions and there was a high rate of invasive mechanical ventilation. ICU, in-hospital and six-month survival were 58.5, 42.0 and $31.2 \%$, respectively. Surgical treatment of lung cancer increased the odds of ICU admission (OR 7.23 (5.14-10.2)). Odds of admission to ICU were reduced with older age (75-80 years OR $0.69(0.49-0.94)$, > 80 years OR 0.21 (0.12-0.37)), female gender (OR $0.73(0.59-0.90)$ ) and radiotherapy (OR 0.54 (0.39-0.73)) or chemotherapy treatment (OR $0.52(0.38-0.70)$ ).

Conclusion: $1.5 \%$ of patients diagnosed with lung cancer are admitted to an ICU but both short term and long term survival was poor. Factors associated with ICU admission included age $<75$ years, male gender and surgical treatment of cancer
\end{abstract}

\section{Background}

Lung cancer is the most common cancer and the leading cause of cancer related death worldwide [1]. Due to the nature of the disease and the aggressive treatments often employed, patients with lung cancer may develop a critical illness such that they require admission to ICU for invasive monitoring or treatment [2]. Of all the individual cancer types, lung cancer has one of the poorest survival after an ICU admission and has been demonstrated

\footnotetext{
* Correspondence: kadunne@doctors.net.uk

${ }^{1}$ Academic department of Anaesthesia, Critical Care and Pain, College of Medical, Veterinary and Life Sciences, University of Glasgow, Glasgow, UK Full list of author information is available at the end of the article
}

to have a high ICU and in-hospital mortality [3-5]. A systematic review of published outcomes for patients with solid tumours admitted to ICU described an average ICU mortality of $40.1 \%$ for patients with lung cancer, the highest of all the individual tumour types described [3]. However, lung cancer is one of the commonest tumour types admitted to ICU $[2,6,7]$.

The proportion of cancer patients that are admitted to ICU is steadily increasing with one US hospital describing a two-fold increase in the number of lung cancer patients admitted to ICU over a ten-year period [8]. A wide variety of factors have been reported to be independently associated with short term mortality, the 
majority being related to the severity of the acute episode of illness requiring critical care and degree of organ dysfunction. However, no previously published studies have attempted to describe which factors are associated with ICU admission for these patients.

The aim of this study was to describe the rate of critical illness resulting in ICU admission among patients with lung cancer and the factors associated with this. We performed a population-based study using linked cancer registry, hospitalisation, ICU audit and death records to determine the risks after a cancer diagnosis.

\section{Methods}

\section{Study design, Population \& Setting}

We conducted a retrospective, observational, multi-centre, population-based study through an analysis of secondary data comprising linked cancer registration, hospital discharge, intensive care and mortality records. Full details are described elsewhere [2]. Patients resident in the West of Scotland who had a diagnosis of lung cancer (ICD-10 codes; C33, C34.0, C34.1, C34.2, C34.3, C34.8 and C34.9) on the Scottish Cancer Registry between 1st January 2000 and 31st December 2009 were included in this study. Small Cell Lung Cancers (SCLC) were classified as ICD-O morphology M 8041/3 to M 8045/9; all others were classified Non-Small Cell Lung Cancers (NSCLC). We investigated whether they had been admitted to one of the 16 general ICUs located in the region within 2 years of the date of cancer incidence up to $31 / 12 / 2011$. The first admission to an ICU within 2 years following a diagnosis of lung cancer was identified and linked to the appropriate individual episode of hospital care. We used death and hospital discharge records to identify whether patients died during their hospital stay.

\section{Data sources and variables}

The study used four linked data sets: the Scottish Cancer Registry, Scottish Morbidity Record 01, national death records and the Scottish Intensive Society Audit Group WardWatcher ICU database. WardWatcher collects data on patient demongraphics, admitting specialty, admission diagnosis, the Acute Physiology and Chronic Health Evaluation (APACHE) II scoring system, and the type of organ support. Organ support was defined as receipt of invasive mechanical ventilation, vasoactive drugs to provide cardiovascular support, or renal replacement therapy.

Socioeconomic status was measured using the Scottish Index for Multiple Deprivation (SIMD 2009 V2 Scotland) quintile, an aggregate measure used to identify small area concentrations of deprivation.

The SMR01 hospital discharge records were reviewed for all primary and secondary diagnoses in the 5 years preceding the date of lung cancer incidence. These ICD9 or ICD-10 coded diagnoses were computed to determine the presence or absence of co-morbid disease according to the Charleson Comorbidity Index (CCI) through previously published coding algorithms $[9,10]$.

This study was approved by the West of Scotland Research and Ethics Committee. Approvals to use the data were obtained from the West of Scotland Critical Care Research Network, SICSAG, and the West of Scotland Cancer Surveillance Unit.

\section{Statistical analyses}

Patients who were admitted to an ICU were compared to the remainder of the incident lung cancer cohort according to demographic, clinical and cancer-related variables. Summary statistics including mean and standard deviation or median and inter-quartile range were determined for continuous exposure variables accordingly. Categorical variables were summarised according to frequencies and proportions. Non-ordered categorical variables were analysed by a chi-squared test of association whilst ordinal variables were compared between the two groups by use of a chi-squared test for trend.

Factors associated with admission to ICU were estimated using a multivariable logistic regression model. The odds of survival were computed for each exposure variable using univariable analyses with $95 \%$ confidence intervals and $P$ values. Variables found to have a potentially statistically significant alteration in the odds of survival $(P<0.3)$ in univariable analyses were included in an adjusted multivariable model. The cancer treatment modalities (surgery, radiotherapy, chemotherapy) were not included in any multivariable model due to potential collinearity with treatment intent. A $P$ value of $<0.05$ was used to identify statistically significant associations in multivariable modelling for ICU admission.

Statistical analyses were performed using StataCorp 2011 (Stata Statistical Software: Release 12).

\section{Availability of data and materials}

The authors do not have permission to share the data used for this article, however, the data can be accessed via application to the Information Services Division of NHS Scotland.

\section{Results}

There were 26,731 incident cases of primary lung cancer diagnosed during the study period of whom 398 patients $(1.5 \%)$ were admitted to an ICU within 2 years of diagnosis. Figure 1 shows the cumulative hazard curve for time to admission to ICU in days according to sex. There appears to be a sharp rise in the hazard of ICU admission within the first 100 days following a diagnosis of lung cancer for both sexes. The median number of days from lung cancer diagnosis to admission was 52 (IQR 0-106). There 


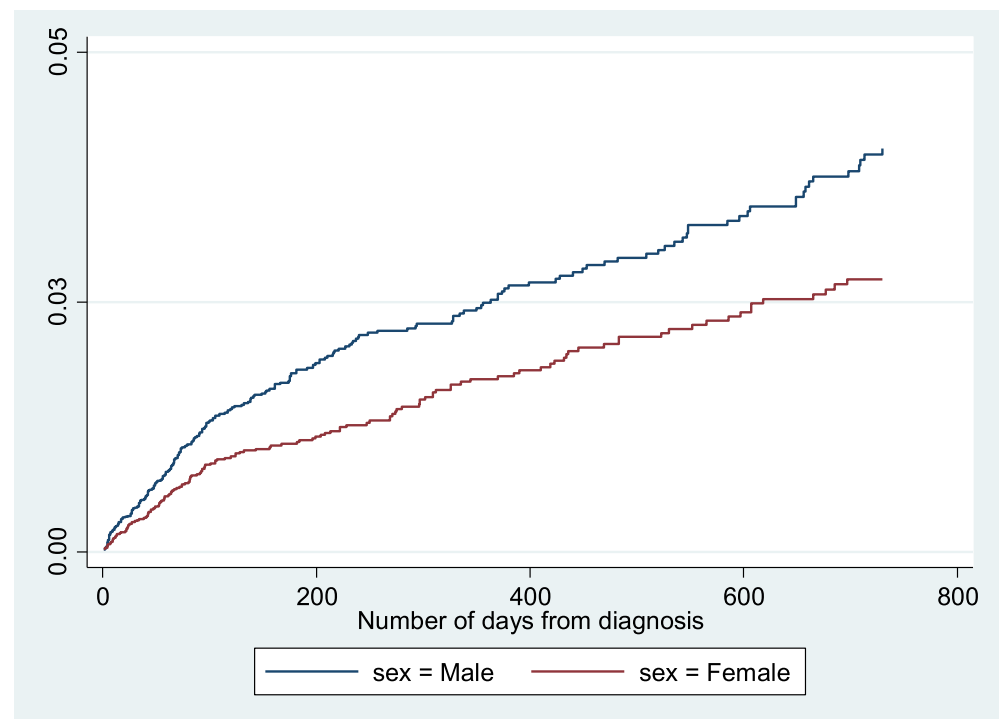

Fig. 1 Cumulative hazard of admission to Intensive Care by time since cancer incidence date according to sex (log-rank test for equality of survivor functions Chi ${ }^{2} 15.22, P<0.001$ )

were fewer ICU admissions amongst women observed than were expected.

Features pertaining to the ICU admission are detailed in Table 1 . The majority of lung cancer patients admitted to ICU were following surgical hospitalisations. Common causes for admission to ICU included lower respiratory tract infection (27.4\%), conditions directly related to lung cancer such as bronchial obstruction or bronchial haemorrhage (20.3\%), and following lung resection surgery (15.3\%). Organ support was frequently utilised with $40.1 \%$ of patients requiring multi-organ support. ICU and hospital median length of stay were 3 (IQR 1-6) and 10 days (IQR 1-19). The proportions of patients who survived ICU, hospital and six-months after hospital discharge were $58.5,42.0$ and $31.2 \%$, respectively.

Table 2 compares the 398 patients admitted to ICU within 2 years of diagnosis to the remaining 26,333 incident primary lung cancer cases. Patients admitted to ICU had a younger age of diagnosis than the non-ICU cohort. There was a larger proportion of males admitted to ICU (61.8\%) than the remainder of the cohort (53.5\%). The largest SIMD quintile represented in each group was the most deprived but deprivation was not associated with admission to ICU. Non-Small Cell Lung cancer was the commonest tumour type in both the ICU and non-ICU groups with a higher proportion in the ICU group (92\% versus $84 \%$ ). Comorbidity was common in both groups (39.9\% ICU admissions vs. $35.8 \%$ of non-ICU admissions, p 0.09). Those admitted to an ICU were more likely to have undergone a surgical treatment for lung cancer with a curative treatment plan. Treatment with radiotherapy or chemotherapy occurred in a higher proportion of the non-ICU population.
Table 3 describes factors associated with ICU admission on univariable and multivariable logistic regression analysis. The largest effect was seen for patients receiving surgical treatment for cancer, with OR 7.23 (95\% CI 5.1410.2) for ICU admission, when compared to patients that did not receive surgical intervention. Unknown treatment intent was associated with ICU admission to a lesser extent (OR 1.54 (95\% CI 1.08-2.20)). Increasing age, female gender and radiotherapy or chemotherapy treatment were all associated with a reduced odds of ICU admission in the multivariable model.

\section{Discussion}

In a large inclusive cohort of lung cancer patients diagnosed over a ten-year period, we found that $1.5 \%$ were admitted to a general ICU within 2 years of diagnosis. ICU admission was greatest in the period shortly after cancer diagnosis and this is likely to reflect patients developing critical illness as a consequence of active cancer or its treatment.

\section{Clinical features in patients with lung cancer admitted to ICU}

Reasons for ICU admission were predominantly related to respiratory conditions with lower respiratory tract infections and complications from the malignancy accounting for 27.4 and $20.3 \%$ of all admissions respectively. However, the ICU admission was entirely unrelated to the lung cancer diagnosis/ treatment in one in four ICU lung cancer patients. The majority of ICU patients with lung cancer required organ support, the most common modality being 
Table 1 Clinical features of Lung cancer patients admitted to ICU

\begin{tabular}{ll}
\hline Categories & $N=368$ \\
& $N(\%)$ \\
\hline
\end{tabular}

Admission Diagnosis

81 (20.3)

Related to lung cancer

$43(10.8)$

Acute cardiac decompensation

$109(27.4)$

Chest infection

$61(15.3)$

Unrelated to lung cancer

$104(26.1)$

Cardiac Arrest Precipitating Admission

Prior CPR

$29(7.3)$

No prior CPR

$369(92.7)$

APACHE II Score

$8-12$

13-17

$20(5.0)$

$67(16.8)$

18-22

77 (19.4)

23-27

$74(18.6)$

$\geq 28$

Missing

$55(13.8)$

$105(26.4)$

Admission Specialty

Medical

134 (33.7)

Surgical

Number of Organs Supported

None

79 (19.9)

1

2

3

Not known

Receipt of Mechanical Ventilation

No

Yes

Not known

Receipt of Renal Replacement Therapy

No

Yes

Not known

Receipt of Vasoactive Agents

No

Yes

Not known

Charlson Co-Morbidity Index

0

$\geq 1$

Length of stay

ICU

Hospital

$25(6.3)$

62 (15.6)

99 (24.9)

282 (70.9)

17 (4.3)

322 (80.9)

$27(6.8)$

$49(12.3)$

$183(46.0)$

$182(45.7)$

$33(8.3)$

239 (60.1)

159 (39.9)

3 days (IQR 1-6)

10 days (IQR 1-19)
$264(66.3)$

Table 1 Clinical features of Lung cancer patients admitted to ICU (Continued)

\begin{tabular}{ll}
\hline Categories & $N=368$ \\
& $N(\%)$ \\
\hline Mortality & \\
ICU & $165(41.5)$ \\
Hospital & $231(58.0)$ \\
Six-month & $274(68.8)$ \\
\hline
\end{tabular}

invasive ventilation (70.9\%) reflecting the high incidence of respiratory disorders.

For patients admitted to ICU, mortality in ICU, hospital and six-months post admission were 41.5, 58.0 and $68.8 \%$, respectively. Mortality in our study was relatively high when compared with previous multi-centre studies [1113]. The largest study of this nature was performed on the American surveillance, epidemiology and end resultsmedicare registry (SEER), including nearly 50,000 patients. Hospital mortality was reported as $24 \%$, significantly lower than that seen in this study. However, mortality at sixmonths was similar at 65\% [13]. This registry-based study did not detail any of the features of the critical illness which has been demonstrated to be the largest determinant of short-term mortality [3]. Soares et al conducted the only prospective, multi-centre cohort study and reported ICU, in-hospital and six-month mortality of 28, 39 and $55 \%$ respectively. However, the proportion of subjects requiring mechanical ventilation was only $53 \%$ compared with the $79 \%$ observed in our study, suggesting that the burden of critical illness was less.(11).

\section{Differences between ICU and non-ICU groups}

While the majority of patients with lung cancer were treated with palliative intent, a disproportionate number of patients in the ICU group had received curative treatment with the majority of patients receiving surgical intervention. This observation likely reflects clinician behaviour whereby patients with curative treatment intent are preferentially selected for ICU during an acute critical illness. However, this could also be partially attributable to postoperative complications in patients undergoing curative surgical procedures as the majority of ICU admissions were surgical in nature (66\%) and ICU admission was directly related to cancer surgery in $15 \%$.

\section{Factors associated with ICU admission in patients with lung cancer}

Surgical treatment of cancer was found to be the strongest predictor of ICU admission with a seven-fold increase in odds of ICU admission compared with those not treated with surgery. This may be due to critical illness occurring at the time of surgery with one in eight ICU admissions were directly attributable to the surgical 
Table 2 Features of lung cancer patients that are and are not admitted to Intensive Care

\begin{tabular}{|c|c|c|c|}
\hline & $\begin{array}{l}\text { ICU Admissions } \\
N=398 \\
\mathrm{n}(\%)\end{array}$ & $\begin{array}{l}\text { Non-admissions } \\
N=26,333 \\
\mathrm{n}(\%)\end{array}$ & $P$ value \\
\hline \multicolumn{4}{|l|}{ Age at Incidence } \\
\hline Mean (SD) & 67 (SD 8.8) & 71 (SD 10.3) & $<0.001$ \\
\hline \multicolumn{4}{|l|}{ Sex } \\
\hline Male & $246(61.8)$ & $14,085(53.5)$ & \multirow[t]{2}{*}{0.001} \\
\hline Female & $152(38.2)$ & $12,248(46.5)$ & \\
\hline \multicolumn{4}{|l|}{ SIMD Quintile } \\
\hline 1 (Most deprived) & $146(36.7)$ & $10,691(40.6)$ & \multirow[t]{5}{*}{$0.350^{\mathrm{a}}$} \\
\hline 2 & $106(26.6)$ & $6692(25.4)$ & \\
\hline 3 & $73(18.3)$ & 4108 (15.6) & \\
\hline 4 & $43(10.8)$ & $2739(10.4)$ & \\
\hline 5 (Most affluent) & $30(7.5)$ & $2103(8.0)$ & \\
\hline \multicolumn{4}{|l|}{ Histology } \\
\hline $\mathrm{SCLC}$ & $32(8.0)$ & $4222(16.0)$ & \multirow[t]{2}{*}{$<0.001$} \\
\hline NSCLC & $366(92.0)$ & $22,111(84.0)$ & \\
\hline \multicolumn{4}{|c|}{ Charlson Co-Morbidity Index } \\
\hline 0 & $239(60.1)$ & $16,894(64.2)$ & \multirow[t]{2}{*}{0.090} \\
\hline$\geq 1$ & $159(39.9)$ & $9439(35.8)$ & \\
\hline \multicolumn{4}{|c|}{ Year of Cancer Incidence } \\
\hline 2000 & $43(10.8)$ & $2562(9.7)$ & \multirow[t]{10}{*}{$0.004^{a}$} \\
\hline 2001 & $39(9.8)$ & $2389(9.1)$ & \\
\hline 2002 & $42(10.6)$ & $2624(10.0)$ & \\
\hline 2003 & $57(14.3)$ & $2600(9.9)$ & \\
\hline 2004 & $35(8.8)$ & $2607(9.9)$ & \\
\hline 2005 & $42(10.6)$ & $2609(9.9)$ & \\
\hline 2006 & $38(9.5)$ & $2738(10.4)$ & \\
\hline 2007 & $46(11.6)$ & $2716(10.3)$ & \\
\hline 2008 & $35(8.8)$ & $2700(10.3)$ & \\
\hline 2009 & $21(5.3)$ & $2788(10.6)$ & \\
\hline \multicolumn{4}{|l|}{ Treated with Surgery } \\
\hline No & $168(42.2)$ & $21,540(81.8)$ & \multirow[t]{3}{*}{$<0.001$} \\
\hline Yes & $210(52.8)$ & $2505(9.5)$ & \\
\hline Not known & $20(5.0)$ & $2288(8.7)$ & \\
\hline \multicolumn{4}{|c|}{ Treated with Radiotherapy } \\
\hline No & $280(70.4)$ & $15,510(58.9)$ & \multirow[t]{3}{*}{$<0.001$} \\
\hline Yes & $53(13.3)$ & 7876 (29.9) & \\
\hline Not known & $65(16.3)$ & $2947(11.2)$ & \\
\hline \multicolumn{4}{|c|}{ Treated with Chemotherapy } \\
\hline No & $283(71.1)$ & $17,342(65.9)$ & \multirow[t]{3}{*}{$<0.001$} \\
\hline Yes & $56(14.1)$ & $6468(24.6)$ & \\
\hline Not known & $59(14.8)$ & $2523(9.6)$ & \\
\hline \multicolumn{4}{|l|}{ Therapy objectives } \\
\hline Curative & $140(35.2)$ & 2481 (9.4) & $<0.001$ \\
\hline
\end{tabular}

Table 2 Features of lung cancer patients that are and are not admitted to Intensive Care (Continued)

\begin{tabular}{llll}
\hline & ICU Admissions \\
$N=398$ & $\begin{array}{l}\text { Non-admissions } \\
\mathrm{n}=26,333 \\
\mathrm{n}(\%)\end{array}$ & $P$ value \\
\hline Palliative & $184(46.2)$ & $20,657(78.5)$ \\
Not known & $74(18.6)$ & $3195(12.1)$ \\
\hline \multicolumn{2}{l}{ SCLC Small cell lung cancer, NSCLC Non-small cell lung cancer }
\end{tabular}

treatment. While it is possible that patients having received curative surgery were being considered better candidates for ICU, treatment intent was not associated with ICU admission on the multivariable model. Other treatment interventions influenced ICU admission by reducing the odds by approximately half in those who were treated with chemotherapy or radiotherapy compared with those who were not. As these patients are being admitted early after diagnosis they may not have had the opportunity to receive these treatment interventions prior to ICU admission.

Age of incidence was found to be a strong negative predictor of ICU admission with a reduction in the odds of admission with increasing age above 75 years that reduces further for those aged over 80 years. Previous work by Azoulay et al. demonstrated that the largest influence on a refusal for ICU admission in critically ill patients with cancer was an age of over 65 years [14]. Within the general ICU population, increasing age has been associated with poorer survival both in the short and longer term, and that this was particularly pronounced for patients aged over 75 years [15]. It seems unlikely that critical illness is less common with increasing age, particularly given the increased incidence of comorbidities encountered in an elderly population, rather that concerns about poor outcomes in the elderly patients swings the balance of benefit versus harm away from ICU admission.

Comorbidity was not associated with an increase in odds of ICU admission in the multivariable model. The study by Slatore et al. [16] demonstrated that comorbidity has been associated with poorer outcome after ICU admission in patients with lung cancer, however, our study suggests that this does not necessarily influence admission patterns.

Females had a statistically significant reduction in the odds of ICU admission compared to males (OR 0.75, 95\% CI 0.61-0.92, $P=0.006$ ). An increased incidence of critical illness in men has been described for patients with severe sepsis and trauma $[17,18]$. The cause of this is not clear but may reflect differences in lifestyle choices, health behaviours or the impact of hormones on stress responses. Furthermore, it is possible that the gender difference may not reflect difference in rates of critical illness but a difference in preferences for end of life care. It is known from other studies that survival from lung cancer is poorer in men than women overall. 
Table 3 Factors associated with ICU admission on univariable and multivariable logistic regression

\begin{tabular}{|c|c|c|c|c|}
\hline & $\begin{array}{l}\text { Univariable } \\
\text { Odds Ratio (95\% Cl) }\end{array}$ & $P$ value & $\begin{array}{l}\text { Multivariable } \\
\text { Odds Ratio }(95 \% \text { Cl) }\end{array}$ & $P$ value \\
\hline \multicolumn{5}{|c|}{ Age at Incidence Quintile* } \\
\hline $15-62$ & 1 & & 1 & \\
\hline $63-69$ & $1.21(0.93-1.57)$ & 0.153 & $1.15(0.88-1.51)$ & 0.294 \\
\hline $70-74$ & $0.90(0.67-1.20)$ & 0.463 & $0.87(0.64-1.17)$ & 0.360 \\
\hline $75-80$ & $0.62(0.46-0.85)$ & 0.003 & $0.69(0.49-0.94)$ & 0.019 \\
\hline$>80$ & $0.16(0.09-0.28)$ & $<0.001$ & $0.21(0.12-0.37)$ & $<0.001$ \\
\hline \multicolumn{5}{|l|}{ Sex } \\
\hline Male & 1 & & 1 & \\
\hline Female & $0.71(0.58-0.87)$ & 0.001 & $0.73(0.59-0.90)$ & 0.003 \\
\hline \multicolumn{5}{|l|}{ SIMD Quintile } \\
\hline 1 (Most deprived) & 1 & & 1 & \\
\hline 2 & $1.16(0.90-1.49)$ & 0.249 & $1.16(0.89-1.50)$ & 0.269 \\
\hline 3 & $1.30(0.98-1.73)$ & 0.068 & $1.37(1.03-1.83)$ & 0.033 \\
\hline 4 & $1.15(0.82-1.62)$ & 0.425 & $1.31(0.92-1.86)$ & 0.132 \\
\hline 5 (Most affluent) & $1.04(0.70-1.55)$ & 0.829 & $1.08(0.69-1.55)$ & 0.696 \\
\hline \multicolumn{5}{|l|}{ Therapy Objectives } \\
\hline Curative & 1 & & 1 & \\
\hline Palliative & $0.16(0.13-0.20)$ & $<0.001$ & $1.08(0.75-1.54)$ & 0.682 \\
\hline Not known & $0.41(0.31-0.55)$ & $<0.001$ & $1.54(1.08-2.20)$ & 0.017 \\
\hline \multicolumn{5}{|l|}{ Treated with Surgery } \\
\hline No & 1 & & 1 & \\
\hline Yes & $10.75(8.7-13.2)$ & $<0.001$ & $7.23(5.14-10.2)$ & $<0.001$ \\
\hline Not known & $1.12(0.70-1.79)$ & 0.631 & $0.50(0.27-0.91)$ & 0.025 \\
\hline \multicolumn{5}{|c|}{ Treated with Radiotherapy } \\
\hline No & 1 & & 1 & \\
\hline Yes & $0.37(0.28-0.50)$ & $<0.001$ & $0.54(0.39-0.73)$ & $<0.001$ \\
\hline Not known & $1.22(0.93-1.60)$ & 0.150 & $1.32(0.78-2.24)$ & 0.298 \\
\hline \multicolumn{5}{|c|}{ Treated with Chemotherapy } \\
\hline No & 1 & & 1 & \\
\hline Yes & $0.53(0.40-0.71)$ & $<0.001$ & $0.52(0.38-0.70)$ & $<0.001$ \\
\hline Not known & $1.43(1.08-1.90)$ & 0.013 & $1.07(0.62-1.84)$ & 0.814 \\
\hline \multicolumn{5}{|c|}{ Charlson Co-morbidity Index } \\
\hline 0 & 1 & & 1 & \\
\hline$\geq 1$ & $1.19(0.97-1.46)$ & 0.091 & $1.22(0.99-1.51)$ & 0.059 \\
\hline
\end{tabular}

Among those with a critical illness that we report, survival was also poorer in men than women. Given that a minority of lung cancer patients is admitted to an ICU $(1.5 \%)$, we would conclude that both non-critically ill as well as critically ill female lung cancer patients have better survival than men.

\section{Strengths and weaknesses}

Our study has a number of strengths. It is the first study to compare lung cancer patients admitted to an ICU with those who were not over a ten-year period. It utilised a large sample size and was conducted across multiple general rather than oncological ICUs, which may improve its generalisability. We used datasets with high levels of case ascertainment for cancer incidence, hospitalisation and deaths. Measurement bias was reduced through the use of objective scoring systems to capture acute illness and past medical history. The use of the SIMD provided a robust, multi-dimensional measure of socioeconomic status rather than relying on a single 
domain as a proxy for affluence as in previous studies $[12,13,19]$. Exposure variables from the ICU admission were collected and determined prospectively thereby reducing the likelihood of misclassification.

This study has several limitations. Large proportions of missing data precluded the use of information on ethnicity, cancer stage and pathological sub-division which could confound the observed associations. Furthermore, while the date of cancer diagnosis in all cases was prior to ICU admission, we could not determine the certainty of diagnosis at the point of entry to critical care which may affect decisions about whether to admit a patient. We accept that approaches to managing critical illness are likely to differ in patients receiving palliative care and while we adjusted for treatment objective (curative, palliative or unknown), we did not have information on palliative care involvement within the data sources utilised in this study.

\section{Conclusion}

Lung cancer is the most common cause of cancer related death worldwide [1]. Our results highlight that only a small proportion of patients with lung cancer were subsequently admitted to an ICU within 2 years of diagnosis (1.5\%). Over half of those who were admitted survived to ICU discharge with nearly a third surviving to six-months from admission. It is unclear why some factors that are usually associated with a better prognosis, such as younger age and surgical treatment, are associated with greater risks of ICU admission. This may represent physician decision making and further prospective research is needed to explore the clinical pathways prior to ICU admission.

\section{Abbreviations \\ APACHE: Acute Physiology and Chronic Health Evaluation; CCl: Charlson Co- morbidity Index; Cl: Confidence Interval; HDU: High Dependency Unit; ICU: Intensive Care Unit; ICD: International Statistical Classification of Diseases and Related Health Problems; NSCLC: Non Small Cell Lung Cancer; OR: Odds Ratio; SCLC: Small Cell Lung Cancer; SICSAG: The Scottish Intensive Care Society Audit Group; SIMD: The Scottish Index for Multiple Deprivation; SMR 01: The Scottish Morbidity Record 01}

\section{Acknowledgements}

We are grateful to the Scottish Intensive Care Society Audit Group and the West of Scotland Cancer Surveillance Unit for approval to use this data. The authors would like to thank the Information Services Division Scotland, the Scottish Intensive Care Society Audit Group and all patients who participated in the creation of the West of Scotland Critical Care and Cancer Patient Database. The authors would also like to thank Cancer Research UK for funding this research.

Notation of Prior Abstract Publication/Presentation: Part of this work was presented as a poster presentation at the National Cancer Research Institute conference in Liverpool, UK on 6-8th November 2017. An abstract was made available via the conference website.

\section{Authors' contributions}

CHG, PM, KP and DSM conceived and designed the work. CHG, PM and BS carried out the acquisition and analysis of data. $\mathrm{CHG}, \mathrm{KP}$ and DSM drafted the work. CHG, PM, BS, KP, TQ, KH, and DSM contributed to interpretation of data and critically revised the work for important intellectual content. The authors agree to be accountable for all aspects of the work in respect of its accuracy and integrity and approve the final version as submitted. KP takes responsibility for the content of the manuscript, including the data and analysis.

\section{Funding}

The work was funded by a grant provided by Cancer Research UK (C47114A / A16662) to DSM. The sponsors played no role in the study design, handling of data, report writing or decision to submit the paper for publication.

\section{Availability of data and materials}

The data is available through application to the NHS Scotland Information Services Division.

\section{Ethics approval and consent to participate}

This study was approved by the West of Scotland Research and Ethics Committee (reference 12/WS/0075). Approvals to use the data were obtained from the West of Scotland Critical Care Research Network, SICSAG and the West of Scotland Cancer Surveillance Unit.

\section{Consent for publication}

All authors approved the final version of the manuscript to be published.

\section{Competing interests}

The authors declare that they have no competing interests.

\section{Author details}

${ }^{1}$ Academic department of Anaesthesia, Critical Care and Pain, College of Medical, Veterinary and Life Sciences, University of Glasgow, Glasgow, UK. ${ }^{2}$ Department of Public Health, College of Medical, Veterinary and Life Sciences, University of Glasgow, Glasgow, UK.

Received: 31 July 2019 Accepted: 30 January 2020

Published online: 07 February 2020

\section{References}

1. Ferlay J, Soerjomataram I, Dikshit R, Eser S, Mathers C, Rebelo M, et al. Cancer incidence and mortality worldwide: sources, methods and major patterns in GLOBOCAN 2012. Int J Cancer. 2015;136(5):E359-86.

2. Puxty K, McLoone P, Quasim T, Sloan B, Kinsella J, Morrison DS. Risk of critical illness among patients with solid cancers: a population-based observational study. JAMA Oncol. 2015;1(8):1078-85.

3. Puxty K, McLoone P, Quasim T, Kinsella J, Morrison D. Survival in solid cancer patients following intensive care unit admission. Intensive Care Med. 2014:40(10):1409-28.

4. Andrejak C, Terzi N, Thielen S, Bergot E, Zalcman G, Charbonneau P, et al. Admission of advanced lung cancer patients to intensive care unit: a retrospective study of 76 patients. BMC Cancer. 2011;11:159.

5. Soares M, Darmon M, Salluh JIF, Ferreira CG, Thiery G, Schlemmer B, et al. Prognosis of lung cancer patients with life-threatening complications. Chest. 2007;131(3):840-6.

6. Martos-Benitez FD, Soto-Garcia A, Gutierrez-Noyola A. Clinical characteristics and outcomes of cancer patients requiring intensive care unit admission: a prospective study. J Cancer Res Clin Oncol. 2018;144(4):717-23.

7. Ostermann M, Ferrando-Vivas P, Gore C, Power S, Harrison D. Characteristics and outcome of Cancer patients admitted to the ICU in England, Wales, and Northern Ireland and National Trends between 1997 and 2013. Crit Care Med. 2017;45(10):1668-76.

8. Sauer CM, Dong J, Celi LA, Ramazzotti D. Improved Survival of Cancer Patients Admitted to the Intensive Care Unit between 2002 and 2011 at a U.S. Teaching Hospital. Cancer Res Treat. 2019;51(3):973-81.

9. Deyo RA, Cherkin DC, Ciol MA. Adapting a clinical comorbidity index for use with ICD-9-CM administrative databases. J Clin Epidemiol. 1992;45(6):613-9.

10. Quan H, Sundararajan V, Halfon P, Fong A, Burnand B, Luthi JC, et al. Coding algorithms for defining comorbidities in ICD-9-CM and ICD-10 administrative data. Med Care. 2005:43(11):1130-9.

11. Soares M, Toffart AC, Timsit JF, Burghi G, Irrazabal C, Pattison N, et al. Intensive care in patients with lung cancer: a multinational study. Ann Oncol. 2014;25(9):1829-35. 
12. Bonomi MR, Smith CB, Mhango G, Wisnivesky JP. Outcomes of elderly patients with stage IIIB-IV non-small cell lung cancer admitted to the intensive care unit. Lung Cancer. 2012;77(3):600-4.

13. Slatore CG, Cecere LM, Letourneau JL, O'Neil ME, Duckart JP, Wiener RS, et al. Intensive care unit outcomes among patients with lung cancer in the surveillance, epidemiology, and end results-medicare registry. J Clin Oncol. 2012;30(14):1686-91.

14. Azoulay E, Pochard F, Chevret S, Vinsonneau C, Garrouste M, Cohen Y, et al. Compliance with triage to intensive care recommendations. Crit Care Med. 2001;29(11):2132-6.

15. Fuchs L, Chronaki CE, Park S, Novack V, Baumfeld Y, Scott D, et al. ICU admission characteristics and mortality rates among elderly and very elderly patients. Intensive Care Med. 2012;38(10):1654-61.

16. Anisoglou S, Asteriou C, Barbetakis N, Kakolyris S, Anastasiadou G, Pnevmatikos I. Outcome of lung cancer patients admitted to the intensive care unit with acute respiratory failure. Hippokratia. 2013;17(1):60-3.

17. Angus DC, Linde-Zwirble WT, Lidicker J, Clermont G, Carcillo J, Pinsky MR. Epidemiology of severe sepsis in the United States: analysis of incidence, outcome, and associated costs of care. Crit Care Med. 2001;29(7):1303-10.

18. Reade MC, Yende S, Angus DC. Revisiting Mars and Venus: understanding gender differences in critical illness. Crit Care. 2011;15(4):180.

19. Sharma G, Freeman J, Zhang D, Goodwin JS. Trends in end-of-life ICU use among older adults with advanced lung cancer. Chest. 2008;133(1):72-8.

\section{Publisher's Note}

Springer Nature remains neutral with regard to jurisdictional claims in published maps and institutional affiliations.

Ready to submit your research? Choose BMC and benefit from:

- fast, convenient online submission

- thorough peer review by experienced researchers in your field

- rapid publication on acceptance

- support for research data, including large and complex data types

- gold Open Access which fosters wider collaboration and increased citations

- maximum visibility for your research: over $100 \mathrm{M}$ website views per year

At $\mathrm{BMC}$, research is always in progress.

Learn more biomedcentral.com/submissions 